\title{
Search for Dark Photons from Supersymmetric Hidden Valleys
}

V. M. Abazov,${ }^{37}$ B. Abbott,${ }^{75}$ M. Abolins,${ }^{65}$ B. S. Acharya,${ }^{30}$ M. Adams,${ }^{51}$ T. Adams,${ }^{49}$ E. Aguilo, ${ }^{6}$ M. Ahsan, ${ }^{59}$ G. D. Alexeev, ${ }^{37}$ G. Alkhazov, ${ }^{41}$ A. Alton, ${ }^{64} *$ G. Alverson, ${ }^{63}$ G. A. Alves, ${ }^{2}$ L. S. Ancu, ${ }^{36}$ T. Andeen, ${ }^{53}$ M. S. Anzelc, ${ }^{53}$ M. Aoki ${ }^{50}$ Y. Arnoud,${ }^{14}$ M. Arov,${ }^{60}$ M. Arthaud ${ }^{18}$ A. Askew ${ }^{49, \dagger}$ B. Asman, ${ }^{42}$ O. Atramentov, ${ }^{49, \dagger}$ C. Avila, ${ }^{8}$

J. BackusMayes, ${ }^{82}$ F. Badaud, ${ }^{13}$ L. Bagby, ${ }^{50}$ B. Baldin,${ }^{50}$ D. V. Bandurin, ${ }^{59}$ S. Banerjee, ${ }^{30}$ E. Barberis,${ }^{63}$ A.-F. Barfuss,${ }^{15}$ P. Bargassa, ${ }^{80}$ P. Baringer, ${ }^{58}$ J. Barreto, ${ }^{2}$ J. F. Bartlett, ${ }^{50}$ U. Bassler, ${ }^{18}$ D. Bauer, ${ }^{44}$ S. Beale, ${ }^{6}$ A. Bean,${ }^{58}$ M. Begalli, ${ }^{3}$ M. Begel,${ }^{73}$ C. Belanger-Champagne, ${ }^{42}$ L. Bellantoni, ${ }^{50}$ A. Bellavance, ${ }^{50}$ J. A. Benitez, ${ }^{65}$ S. B. Beri, ${ }^{28}$ G. Bernardi, ${ }^{17}$ R. Bernhard, ${ }^{23}$ I. Bertram ${ }^{43}$ M. Besançon, ${ }^{18}$ R. Beuselinck, ${ }^{44}$ V. A. Bezzubov, ${ }^{40}$ P. C. Bhat, ${ }^{50}$ V. Bhatnagar,${ }^{28}$ G. Blazey, ${ }^{52}$ S. Blessing ${ }^{49}$ K. Bloom,${ }^{67}$ A. Boehnlein, ${ }^{50}$ D. Boline, ${ }^{62}$ T. A. Bolton, ${ }^{59}$ E. E. Boos,${ }^{39}$ G. Borissov, ${ }^{43}$ T. Bose,${ }^{62}$ A. Brandt, ${ }^{78}$ R. Brock, ${ }^{65}$ G. Brooijmans, ${ }^{70}$ A. Bross,${ }^{50}$ D. Brown, ${ }^{19}$ X. B. Bu, ${ }^{7}$ D. Buchholz,${ }^{53}$ M. Buehler, ${ }^{81}$ V. Buescher, ${ }^{22}$ V. Bunichev ${ }^{39}$ S. Burdin, ${ }^{43, \$}$ T. H. Burnett, ${ }^{82}$ C. P. Buszello, ${ }^{44}$ P. Calfayan, ${ }^{26}$ B. Calpas, ${ }^{15}$ S. Calvet, ${ }^{16}$ J. Cammin, ${ }^{71}$ M. A. Carrasco-Lizarraga, ${ }^{34}$ E. Carrera, ${ }^{49}$ W. Carvalho, ${ }^{3}$ B. C. K. Casey, ${ }^{50}$ H. Castilla-Valdez, ${ }^{34}$ S. Chakrabarti, ${ }^{72}$ D. Chakraborty, ${ }^{52}$ K. M. Chan, ${ }^{55}$ A. Chandra ${ }^{48}$ E. Cheu, ${ }^{46}$ D. K. Cho, ${ }^{62}$ S. Choi, ${ }^{33}$ B. Choudhary,${ }^{29}$ T. Christoudias, ${ }^{44}$ S. Cihangir, ${ }^{50}$ D. Claes, ${ }^{67}$ J. Clutter, ${ }^{58}$ M. Cooke, ${ }^{50}$ W. E. Cooper, ${ }^{50}$ M. Corcoran, ${ }^{80}$ F. Couderc, ${ }^{18}$ M.-C. Cousinou, ${ }^{15}$ S. Crépé-Renaudin, ${ }^{14}$ V. Cuplov, ${ }^{59}$ D. Cutts, ${ }^{77}$ M. Ćwiok, ${ }^{31}$ A. Das, ${ }^{46}$ G. Davies, ${ }^{44}$ K. De, ${ }^{78}$ S. J. de Jong, ${ }^{36}$ E. De La Cruz-Burelo, ${ }^{34}$ K. DeVaughan, ${ }^{67}$ F. Déliot, ${ }^{18}$ M. Demarteau, ${ }^{50}$ R. Demina,${ }^{71}$ D. Denisov,${ }^{50}$ S. P. Denisov, ${ }^{40}$ S. Desai,${ }^{50}$ H. T. Diehl,${ }^{50}$ M. Diesburg, ${ }^{50}$ A. Dominguez, ${ }^{67}$ T. Dorland, ${ }^{82}$ A. Dubey,${ }^{29}$ L. V. Dudko, ${ }^{39}$ L. Duflot,${ }^{16}$ D. Duggan, ${ }^{49}$ A. Duperrin, ${ }^{15}$ S. Dutt, ${ }^{28}$ A. Dyshkant,${ }^{52}$ M. Eads, ${ }^{67}$ D. Edmunds,${ }^{65}$ J. Ellison, ${ }^{48}$ V. D. Elvira,${ }^{50}$ Y. Enari, ${ }^{77}$ S. Eno,${ }^{61}$ P. Ermolov, ${ }^{39, *}$ M. Escalier,${ }^{15}$ H. Evans,${ }^{54}$ A. Evdokimov, ${ }^{73}$ V. N. Evdokimov, ${ }^{40}$ G. Facini, ${ }^{63}$ A. V. Ferapontov, ${ }^{59}$ T. Ferbel, ${ }^{61,71}$ F. Fiedler, ${ }^{25}$ F. Filthaut ${ }^{36}$ W. Fisher, ${ }^{50}$ H. E. Fisk, ${ }^{50}$ M. Fortner ${ }^{52}$ H. Fox,${ }^{43}$ S. Fu,${ }^{50}$ S. Fuess, ${ }^{50}$ T. Gadfort,${ }^{70}$ C. F. Galea ${ }^{36}$ A. Garcia-Bellido, ${ }^{71}$ V. Gavrilov, ${ }^{38}$ P. Gay,${ }^{13}$ W. Geist,${ }^{19}$ W. Geng, ${ }^{15,65}$ C. E. Gerber, ${ }^{51}$ Y. Gershtein, ${ }^{49, \dagger}$ D. Gillberg, ${ }^{6}$ G. Ginther ${ }^{50,71}$ B. Gómez, ${ }^{8}$ A. Goussiou, ${ }^{82}$ P. D. Grannis, ${ }^{72}$ S. Greder, ${ }^{19}$ H. Greenlee, ${ }^{50}$ Z. D. Greenwood, ${ }^{60}$ E. M. Gregores, ${ }^{4}$ G. Grenier, ${ }^{20}$ Ph. Gris,${ }^{13}$ J.-F. Grivaz, ${ }^{16}$ A. Grohsjean, ${ }^{26}$ S. Grünendahl,${ }^{50}$ M. W. Grünewald, ${ }^{31}$ F. Guo ${ }^{72}$ J. Guo, ${ }^{72}$ G. Gutierrez, ${ }^{50}$ P. Gutierrez,${ }^{75}$ A. Haas, ${ }^{70}$ N. J. Hadley, ${ }^{61}$ P. Haefner,${ }^{26}$ S. Hagopian, ${ }^{49}$ J. Haley, ${ }^{68}$ I. Hall, ${ }^{65}$ R. E. Hall, ${ }^{47}$ L. Han, ${ }^{7}$ K. Harder, ${ }^{45}$ A. Harel, ${ }^{71}$ J. M. Hauptman, ${ }^{57}$ J. Hays, ${ }^{44}$ T. Hebbeker, ${ }^{21}$ D. Hedin, ${ }^{52}$ J. G. Hegeman, ${ }^{35}$

A. P. Heinson, ${ }^{48}$ U. Heintz, ${ }^{62}$ C. Hensel, ${ }^{24}$ I. Heredia-De La Cruz,${ }^{34}$ K. Herner, ${ }^{64}$ G. Hesketh, ${ }^{63}$ M. D. Hildreth, ${ }^{55}$

R. Hirosky, ${ }^{81}$ T. Hoang, ${ }^{49}$ J. D. Hobbs, ${ }^{72}$ B. Hoeneisen, ${ }^{12}$ M. Hohlfeld, ${ }^{22}$ S. Hossain, ${ }^{75}$ P. Houben, ${ }^{35}$ Y. Hu, ${ }^{72}$ Z. Hubacek, ${ }^{10}$ N. Huske, ${ }^{17}$ V. Hynek, ${ }^{10}$ I. Iashvili, ${ }^{69}$ R. Illingworth, ${ }^{50}$ A. S. Ito, ${ }^{50}$ S. Jabeen, ${ }^{62}$ M. Jaffré, ${ }^{16}$ S. Jain, ${ }^{75}$ K. Jakobs, ${ }^{23}$ D. Jamin, ${ }^{15}$ C. Jarvis, ${ }^{61}$ R. Jesik, ${ }^{44}$ K. Johns,${ }^{46}$ C. Johnson, ${ }^{70}$ M. Johnson, ${ }^{50}$ D. Johnston, ${ }^{67}$ A. Jonckheere,${ }^{50}$ P. Jonsson, ${ }^{44}$ A. Juste, ${ }^{50}$ E. Kajfasz, ${ }^{15}$ D. Karmanov, ${ }^{39}$ P. A. Kasper, ${ }^{50}$ I. Katsanos, ${ }^{67}$ V. Kaushik, ${ }^{78}$ R. Kehoe, ${ }^{79}$ S. Kermiche, ${ }^{15}$ N. Khalatyan, ${ }^{50}$ A. Khanov, ${ }^{76}$ A. Kharchilava, ${ }^{69}$ Y. N. Kharzheev, ${ }^{37}$ D. Khatidze,${ }^{70}$ T. J. Kim,${ }^{32}$ M. H. Kirby, ${ }^{53}$ M. Kirsch,${ }^{21}$ B. Klima, ${ }^{50}$ J. M. Kohli, ${ }^{28}$ J.-P. Konrath, ${ }^{23}$ A. V. Kozelov, ${ }^{40}$ J. Kraus,${ }^{65}$ T. Kuhl,,${ }^{25}$ A. Kumar, ${ }^{69}$ A. Kupco, ${ }^{11}$ T. Kurča, ${ }^{20}$ V. A. Kuzmin, ${ }^{39}$ J. Kvita, ${ }^{9}$ F. Lacroix,${ }^{13}$ D. Lam,${ }^{55}$ S. Lammers,${ }^{54}$ G. Landsberg, ${ }^{77}$ P. Lebrun, ${ }^{20}$ W. M. Lee, ${ }^{50}$ A. Leflat,${ }^{39}$ J. Lellouch, ${ }^{17}$ J. Li ${ }^{78,}$ L. Li, ${ }^{48}$ Q. Z. Li, ${ }^{50}$ S. M. Lietti, ${ }^{5}$ J. K. Lim, ${ }^{32}$ D. Lincoln, ${ }^{50}$ J. Linnemann, ${ }^{65}$ V. V. Lipaev, ${ }^{40}$ R. Lipton, ${ }^{50}$ Y. Liu, ${ }^{7}$ Z. Liu, ${ }^{6}$ A. Lobodenko, ${ }^{41}$ M. Lokajicek, ${ }^{11}$ P. Love, ${ }^{43}$ H. J. Lubatti, ${ }^{82}$ R. Luna-Garcia, ${ }^{34,}$ A. L. Lyon, ${ }^{50}$ A. K. A. Maciel, ${ }^{2}$ D. Mackin, ${ }^{80}$ P. Mättig, ${ }^{27}$ A. Magerkurth, ${ }^{64}$ P. K. Mal, ${ }^{82}$ H. B. Malbouisson, ${ }^{3}$ S. Malik, ${ }^{67}$ V.L. Malyshev, ${ }^{37}$ Y. Maravin, ${ }^{59}$ B. Martin, ${ }^{14}$ R. McCarthy, ${ }^{72}$ C. L. McGivern, ${ }^{58}$ M. M. Meijer, ${ }^{36}$ A. Melnitchouk, ${ }^{66}$ L. Mendoza, ${ }^{8}$ D. Menezes, ${ }^{52}$ P. G. Mercadante,${ }^{5}$ M. Merkin, ${ }^{39}$ K. W. Merritt, ${ }^{50}$ A. Meyer, ${ }^{21}$ J. Meyer, ${ }^{24}$ J. Mitrevski, ${ }^{70}$ R. K. Mommsen, ${ }^{45}$ N. K. Mondal,${ }^{30}$ R. W. Moore, ${ }^{6}$ T. Moulik, ${ }^{58}$ G. S. Muanza, ${ }^{15}$ M. Mulhearn, ${ }^{70}$ O. Mundal, ${ }^{22}$ L. Mundim, ${ }^{3}$ E. Nagy, ${ }^{15}$ M. Naimuddin, ${ }^{50}$ M. Narain, ${ }^{77}$ H. A. Neal, ${ }^{64}$ J. P. Negret, ${ }^{8}$ P. Neustroev, ${ }^{41}$ H. Nilsen, ${ }^{23}$ H. Nogima, ${ }^{3}$ S. F. Novaes,${ }^{5}$ T. Nunnemann, ${ }^{26}$ G. Obrant,${ }^{41}$ C. Ochando,${ }^{16}$ D. Onoprienko, ${ }^{59}$ J. Orduna, ${ }^{34}$ N. Oshima, ${ }^{50}$ N. Osman, ${ }^{44}$ J. Osta, ${ }^{55}$ R. Otec, ${ }^{10}$ G. J. Otero y Garzón, ${ }^{1}$ M. Owen, ${ }^{45}$ M. Padilla ${ }^{48}$ P. Padley,${ }^{80}$ M. Pangilinan, ${ }^{77}$ N. Parashar, ${ }^{56}$ S.-J. Park,${ }^{24}$ S. K. Park, ${ }^{32}$ J. Parsons,${ }^{70}$ R. Partridge,${ }^{77}$ N. Parua,${ }^{54}$ A. Patwa ${ }^{73}$ G. Pawloski,${ }^{80}$ B. Penning ${ }^{23}$ M. Perfilov,${ }^{39}$ K. Peters, ${ }^{45}$ Y. Peters, ${ }^{45}$ P. Pétroff, ${ }^{16}$ R. Piegaia, ${ }^{1}$ J. Piper, ${ }^{65}$ M.-A. Pleier, ${ }^{22}$ P. L. M. Podesta-Lerma, ${ }^{34, \|}$ V. M. Podstavkov, ${ }^{50}$ Y. Pogorelov, ${ }^{55}$ M.-E. Pol, ${ }^{2}$ P. Polozov, ${ }^{38}$ A. V. Popov, ${ }^{40}$ C. Potter, ${ }^{6}$ W. L. Prado da Silva,${ }^{3}$

S. Protopopescu, ${ }^{73}$ J. Qian, ${ }^{64}$ A. Quadt,${ }^{24}$ B. Quinn, ${ }^{66}$ A. Rakitine, ${ }^{43}$ M. S. Rangel, ${ }^{16}$ K. Ranjan, ${ }^{29}$ P. N. Ratoff, ${ }^{43}$

P. Renkel, ${ }^{79}$ P. Rich,${ }^{45}$ M. Rijssenbeek,${ }^{72}$ I. Ripp-Baudot, ${ }^{19}$ F. Rizatdinova, ${ }^{76}$ S. Robinson, ${ }^{44}$ R. F. Rodrigues, ${ }^{3}$ M. Rominsky, ${ }^{75}$ C. Royon, ${ }^{18}$ P. Rubinov, ${ }^{50}$ R. Ruchti, ${ }^{55}$ G. Safronov, ${ }^{38}$ G. Sajot, ${ }^{14}$ A. Sánchez-Hernández, ${ }^{34}$ M. P. Sanders, ${ }^{17}$ B. Sanghi, ${ }^{50}$ G. Savage,${ }^{50}$ L. Sawyer,${ }^{60}$ T. Scanlon, ${ }^{44}$ D. Schaile,${ }^{26}$ R. D. Schamberger,${ }^{72}$ Y. Scheglov, ${ }^{41}$ 
H. Schellman, ${ }^{53}$ T. Schliephake, ${ }^{27}$ S. Schlobohm, ${ }^{82}$ C. Schwanenberger, ${ }^{45}$ R. Schwienhorst, ${ }^{65}$ J. Sekaric,${ }^{49}$ H. Severini, ${ }^{75}$ E. Shabalina, ${ }^{24}$ M. Shamim, ${ }^{59}$ V. Shary, ${ }^{18}$ A. A. Shchukin,${ }^{40}$ R. K. Shivpuri ${ }^{29}$ V. Siccardi, ${ }^{19}$ V. Simak, ${ }^{10}$ V. Sirotenko, ${ }^{50}$ P. Skubic, ${ }^{75}$ P. Slattery, ${ }^{71}$ D. Smirnov, ${ }^{55}$ G. R. Snow, ${ }^{67}$ J. Snow, ${ }^{74}$ S. Snyder, ${ }^{73}$ S. Söldner-Rembold, ${ }^{45}$ L. Sonnenschein, ${ }^{21}$ A. Sopczak, ${ }^{43}$ M. Sosebee, ${ }^{78}$ K. Soustruznik, ${ }^{9}$ B. Spurlock, ${ }^{78}$ J. Stark, ${ }^{14}$ V. Stolin, ${ }^{38}$ D. A. Stoyanova, ${ }^{40}$ J. Strandberg, ${ }^{64}$ S. Strandberg, ${ }^{42}$ M. A. Strang, ${ }^{69}$ E. Strauss, ${ }^{72}$ M. Strauss, ${ }^{75}$ R. Ströhmer,${ }^{26}$ D. Strom,${ }^{53}$ L. Stutte,${ }^{50}$ S. Sumowidagdo, ${ }^{49}$

P. Svoisky, ${ }^{36}$ M. Takahashi, ${ }^{45}$ A. Tanasijczuk, ${ }^{1}$ W. Taylor, ${ }^{6}$ B. Tiller,${ }^{26}$ F. Tissandier, ${ }^{13}$ M. Titov, ${ }^{18}$ V. V. Tokmenin, ${ }^{37}$

I. Torchiani, ${ }^{23}$ D. Tsybychev, ${ }^{72}$ B. Tuchming, ${ }^{18}$ C. Tully, ${ }^{68}$ P. M. Tuts, ${ }^{70}$ R. Unalan,,${ }^{65}$ L. Uvarov, ${ }^{41}$ S. Uvarov, ${ }^{41}$

S. Uzunyan, ${ }^{52}$ B. Vachon, ${ }^{6}$ P. J. van den Berg, ${ }^{35}$ R. Van Kooten, ${ }^{54}$ W. M. van Leeuwen, ${ }^{35}$ N. Varelas, ${ }^{51}$ E. W. Varnes ${ }^{46}$

I. A. Vasilyev,${ }^{40}$ P. Verdier, ${ }^{20}$ L. S. Vertogradov ${ }^{37}$ M. Verzocchi, ${ }^{50}$ D. Vilanova, ${ }^{18}$ P. Vint,${ }^{44}$ P. Vokac, ${ }^{10}$ M. Voutilainen, ${ }^{67, \pi}$

R. Wagner, ${ }^{68}$ H. D. Wahl, ${ }^{49}$ M. H. L. S. Wang, ${ }^{71}$ J. Warchol,${ }^{55}$ G. Watts, ${ }^{82}$ M. Wayne, ${ }^{55}$ G. Weber, ${ }^{25}$ M. Weber, ${ }^{50}, * *$

L. Welty-Rieger, ${ }^{54}$ A. Wenger, ${ }^{23, \dagger}$ M. Wetstein, ${ }^{61}$ A. White, ${ }^{78}$ D. Wicke, ${ }^{25}$ M. R. J. Williams, ${ }^{43}$ G. W. Wilson, ${ }^{58}$

S. J. Wimpenny, ${ }^{48}$ M. Wobisch, ${ }^{60}$ D. R. Wood, ${ }^{63}$ T. R. Wyatt ${ }^{45}$ Y. Xie, ${ }^{77}$ C. Xu,${ }^{64}$ S. Yacoob,${ }^{53}$ R. Yamada,${ }^{50}$ W.-C. Yang, ${ }^{45}$ T. Yasuda ${ }^{50}$ Y. A. Yatsunenko, ${ }^{37}$ Z. Ye,${ }^{50}$ H. Yin, ${ }^{7}$ K. Yip ${ }^{73}$ H. D. Yoo, ${ }^{77}$ S. W. Youn, ${ }^{53}$ J. Yu, ${ }^{78}$ C. Zeitnitz, ${ }^{27}$ S. Zelitch, ${ }^{81}$ T. Zhao, ${ }^{82}$ B. Zhou, ${ }^{64}$ J. Zhu, ${ }^{72}$ M. Zielinski, ${ }^{71}$ D. Zieminska, ${ }^{54}$ L. Zivkovic, ${ }^{70}$ V. Zutshi, ${ }^{52}$ and E. G. Zverev ${ }^{39}$

\title{
(D0 Collaboration)
}

\author{
${ }^{1}$ Universidad de Buenos Aires, Buenos Aires, Argentina \\ ${ }^{2}$ LAFEX, Centro Brasileiro de Pesquisas Físicas, Rio de Janeiro, Brazil \\ ${ }^{3}$ Universidade do Estado do Rio de Janeiro, Rio de Janeiro, Brazil \\ ${ }^{4}$ Universidade Federal do ABC, Santo André, Brazil \\ ${ }^{5}$ Instituto de Física Teórica, Universidade Estadual Paulista, São Paulo, Brazil \\ ${ }^{6}$ University of Alberta, Edmonton, Alberta, Canada; \\ Simon Fraser University, Burnaby, British Columbia, Canada; \\ York University, Toronto, Ontario, Canada \\ and McGill University, Montreal, Quebec, Canada \\ ${ }^{7}$ University of Science and Technology of China, Hefei, People's Republic of China \\ ${ }^{8}$ Universidad de los Andes, Bogotá, Colombia \\ ${ }^{9}$ Center for Particle Physics, Charles University, Faculty of Mathematics and Physics, Prague, Czech Republic \\ ${ }^{10}$ Czech Technical University in Prague, Prague, Czech Republic \\ ${ }^{11}$ Center for Particle Physics, Institute of Physics, Academy of Sciences of the Czech Republic, Prague, Czech Republic \\ ${ }^{12}$ Universidad San Francisco de Quito, Quito, Ecuador \\ ${ }^{13}$ LPC, Université Blaise Pascal, CNRS/IN2P3, Clermont, France \\ ${ }^{14}$ LPSC, Université Joseph Fourier Grenoble 1, CNRS/IN2P3, Institut National Polytechnique de Grenoble, Grenoble, France \\ ${ }^{15}$ CPPM, Aix-Marseille Université, CNRS/IN2P3, Marseille, France \\ ${ }^{16}$ LAL, Université Paris-Sud, IN2P3/CNRS, Orsay, France \\ ${ }^{17}$ LPNHE, IN2P3/CNRS, Universités Paris VI and VII, Paris, France \\ ${ }^{18}$ CEA, Irfu, SPP, Saclay, France \\ ${ }^{19}$ IPHC, Université de Strasbourg, CNRS/IN2P3, Strasbourg, France \\ ${ }^{20} I P N L$, Université Lyon 1, CNRS/IN2P3, Villeurbanne, France and Université de Lyon, Lyon, France \\ ${ }^{21}$ III. Physikalisches Institut A, RWTH Aachen University, Aachen, Germany \\ ${ }^{22}$ Physikalisches Institut, Universität Bonn, Bonn, Germany \\ ${ }^{23}$ Physikalisches Institut, Universität Freiburg, Freiburg, Germany \\ ${ }^{24}$ II. Physikalisches Institut, Georg-August-Universität Göttingen, Göttingen, Germany \\ ${ }^{25}$ Institut für Physik, Universität Mainz, Mainz, Germany \\ ${ }^{26}$ Ludwig-Maximilians-Universität München, München, Germany \\ ${ }^{27}$ Fachbereich Physik, University of Wuppertal, Wuppertal, Germany \\ ${ }^{28}$ Panjab University, Chandigarh, India \\ ${ }^{29}$ Delhi University, Delhi, India \\ ${ }^{30}$ Tata Institute of Fundamental Research, Mumbai, India \\ ${ }^{31}$ University College Dublin, Dublin, Ireland \\ ${ }^{32}$ Korea Detector Laboratory, Korea University, Seoul, Korea \\ ${ }^{33}$ SungKyunKwan University, Suwon, Korea \\ ${ }^{34}$ CINVESTAV, Mexico City, Mexico \\ ${ }^{35}$ FOM-Institute NIKHEF and University of Amsterdam/NIKHEF, Amsterdam, The Netherlands \\ ${ }^{36}$ Radboud University Nijmegen/NIKHEF, Nijmegen, The Netherlands \\ ${ }^{37}$ Joint Institute for Nuclear Research, Dubna, Russia
}


${ }^{38}$ Institute for Theoretical and Experimental Physics, Moscow, Russia

${ }^{39}$ Moscow State University, Moscow, Russia

${ }^{40}$ Institute for High Energy Physics, Protvino, Russia

${ }^{41}$ Petersburg Nuclear Physics Institute, St. Petersburg, Russia

${ }^{42}$ Stockholm University, Stockholm, Sweden,

and Uppsala University, Uppsala, Sweden

${ }^{43}$ Lancaster University, Lancaster, United Kingdom

${ }^{44}$ Imperial College, London, United Kingdom

${ }^{45}$ University of Manchester, Manchester, United Kingdom

${ }^{46}$ University of Arizona, Tucson, Arizona 85721, USA

${ }^{47}$ California State University, Fresno, California 93740, USA

${ }^{48}$ University of California, Riverside, California 92521, USA

${ }^{49}$ Florida State University, Tallahassee, Florida 32306, USA

${ }^{50}$ Fermi National Accelerator Laboratory, Batavia, Illinois 60510, USA

${ }^{51}$ University of Illinois at Chicago, Chicago, Illinois 60607, USA

${ }^{52}$ Northern Illinois University, DeKalb, Illinois 60115, USA

${ }^{53}$ Northwestern University, Evanston, Illinois 60208, USA

${ }^{54}$ Indiana University, Bloomington, Indiana 47405, USA

${ }^{55}$ University of Notre Dame, Notre Dame, Indiana 46556, USA

${ }^{56}$ Purdue University Calumet, Hammond, Indiana 46323, USA

${ }^{57}$ Iowa State University, Ames, Iowa 50011, USA

${ }^{58}$ University of Kansas, Lawrence, Kansas 66045, USA

${ }^{59}$ Kansas State University, Manhattan, Kansas 66506, USA

${ }^{60}$ Louisiana Tech University, Ruston, Louisiana 71272, USA

${ }^{61}$ University of Maryland, College Park, Maryland 20742, USA

${ }^{62}$ Boston University, Boston, Massachusetts 02215, USA

${ }^{63}$ Northeastern University, Boston, Massachusetts 02115, USA

${ }^{64}$ University of Michigan, Ann Arbor, Michigan 48109, USA

${ }^{65}$ Michigan State University, East Lansing, Michigan 48824, USA

${ }^{66}$ University of Mississippi, University, Mississippi 38677, USA

${ }^{67}$ University of Nebraska, Lincoln, Nebraska 68588, USA

${ }^{68}$ Princeton University, Princeton, New Jersey 08544, USA

${ }^{69}$ State University of New York, Buffalo, New York 14260, USA

${ }^{70}$ Columbia University, New York, New York 10027, USA

${ }^{71}$ University of Rochester, Rochester, New York 14627, USA

${ }^{72}$ State University of New York, Stony Brook, New York 11794, USA

${ }^{73}$ Brookhaven National Laboratory, Upton, New York 11973, USA

${ }^{74}$ Langston University, Langston, Oklahoma 73050, USA

${ }^{75}$ University of Oklahoma, Norman, Oklahoma 73019, USA

${ }^{76}$ Oklahoma State University, Stillwater, Oklahoma 74078, USA

${ }^{77}$ Brown University, Providence, Rhode Island 02912, USA

${ }^{78}$ University of Texas, Arlington, Texas 76019, USA

${ }^{79}$ Southern Methodist University, Dallas, Texas 75275, USA

${ }^{80}$ Rice University, Houston, Texas 77005, USA

${ }^{81}$ University of Virginia, Charlottesville, Virginia 22901, USA

${ }^{82}$ University of Washington, Seattle, Washington 98195, USA

(Received 11 May 2009; published 17 August 2009)

We search for a new light gauge boson, a dark photon, with the D0 experiment. In the model we consider, supersymmetric partners are pair produced and cascade to the lightest neutralinos that can decay into the hidden sector state plus either a photon or a dark photon. The dark photon decays through its mixing with a photon into fermion pairs. We therefore investigate a previously unexplored final state that contains a photon, two spatially close leptons, and large missing transverse energy. We do not observe any evidence for dark photons and set a limit on their production.

DOI: 10.1103/PhysRevLett.103.081802

Hidden valley models [1] introduce a new hidden sector, which is very weakly coupled to the standard model (SM) particles, and therefore can easily escape detection. An important subset of hidden valley models also contain
PACS numbers: 14.80.Ly, 12.60.Jv, 13.85.Qk, 95.35.+d

supersymmetry (SUSY), a fundamental symmetry between fermions and bosons postulating the existence of SUSY partners. At colliders, in the case of $R$-parity conservation [2], superpartners are produced in pairs and decay to the 
SM particles and the lightest superpartner (LSP). The LSP is a stable, weakly interacting particle, and cannot be detected in collider detectors.

Recently, these models were called upon to explain the results of several cosmic ray detection experiments $[3,4]$. Taken together with other experiments, including new results from Fermi/LAT [5], there is evidence of an excess of high energy positrons and no excessive production of antiprotons or photons. The excess can be attributed [6] to the dark matter particles annihilating into pairs of new light gauge bosons, dark photons, which are force carriers in the hidden sector. The dark photon mass cannot be much larger than $1 \mathrm{GeV}$ to give rise to Sommerfeld enhancement [7] of the dark matter annihilation cross section, and not to decay into neutral pions and/or baryons. The masses of the hidden sector states are also around $1 \mathrm{GeV}$, with mass splitting around $\mathrm{MeV}$, thus providing a possible explanation of the DAMA experiment [8] signal through "inelastic dark matter" scenarios. Dark photons decay through mixing with photons into SM fermions with branching fractions that can be calculated from the measurements [9] of $R=$ $\sigma\left(e^{+} e^{-} \rightarrow\right.$ hadrons $) / \sigma\left(e^{+} e^{-} \rightarrow \mu^{+} \mu^{-}\right)$, and strongly depend on the dark photon mass. For dark photon masses $\left(m_{\gamma_{D}}\right)$ below the dimuon threshold of $\simeq 200 \mathrm{MeV}$, only decays into electrons are possible. For $m_{\gamma_{D}} \simeq 0.5 \mathrm{GeV}$ the decay rates into electrons and muons are approximately $40 \%$ each. The lowest value of the leptonic branching $(3.7 \%)$ occurs if the dark photon mass is accidentally equal to that of the $\phi$ meson.

In this Letter we will follow the phenomenological scenario developed in [10]. A diagram of a possible process at the Fermilab Tevatron Collider is shown in Fig. 1. Gauginos are pair produced and decay into SM particles and the lightest neutral gaugino (neutralino, $\tilde{\chi}_{1}^{0}$ ), which in turn decays with comparable branching ratios into either a hidden sector dark neutralino $\tilde{X}$ (which is the LSP), and a photon, or into dark neutralino and a dark photon $\left(\gamma_{D}\right)$.

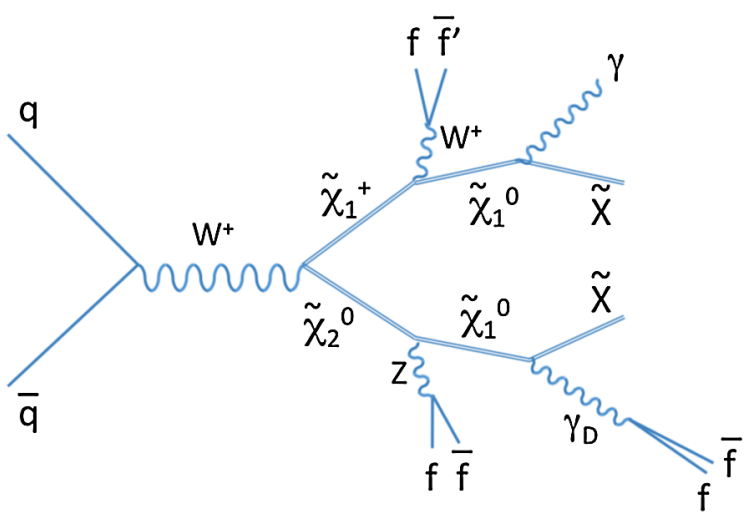

FIG. 1 (color online). One of the diagrams giving rise to the events with a photon, dark photon $\left(\gamma_{D}\right)$, and large missing energy due to escaping dark neutralinos $(\tilde{X})$ at the Fermilab Tevatron Collider.
Hadronic dark photon decays are overwhelmed by SM jet backgrounds. Thus, we only consider dark photon decays into isolated electron or muon pairs. Both dark neutralinos escape detection and result in large missing transverse energy $\left(\mathscr{E}_{T}\right)$. The branching fraction of the neutralino into the dark photon, $\mathcal{B}=\operatorname{Br}\left(\tilde{\chi}_{1}^{0} \rightarrow \gamma_{D} \tilde{X}\right)$, is a free parameter of the model. If it is small, the decays into a photon dominate, and signature is the same as of SUSY with gauge-mediated breaking [11] with the neutralino as next-to-lightest superpartner (NLSP). Larger values of $\mathcal{B}$ give rise to events where one of the two neutralinos decays into a dark photon, resulting in a final state with one photon, two spatially close (and therefore not satisfying traditional isolation requirements) leptons and large $\mathscr{E}_{T}$.

This Letter describes a search for this, so far unexplored, final state in $p \bar{p}$ collisions at a center of mass energy of $1.96 \mathrm{TeV}$ recorded by the D0 detector [12] at the Fermilab Tevatron Collider. As is described below, our search is optimized for low dark photon masses, $m_{\gamma_{D}}<2.5 \mathrm{GeV}$. We consider prompt dark photon decays. Although the experimental analysis is sensitive to macroscopic lifetimes, the expectation is that the neutralino decays to a photon are negligible for small couplings between photon and a dark photon [10], making this channel unfavorable for searches for long-lived dark photons. Another theoretical scenario is the case where the neutralino decays into a hidden state $\tilde{Y}$ with somewhat higher mass than the dark neutralino. The $\tilde{Y}$ may cascade down to the dark neutralino through other hidden states which may be long-lived and can result in the emission of highly collimated low energy SM particles, some of which could be leptons. Most of the energy of the $\tilde{Y}$ will stay in the hidden sector and therefore the high $\mathscr{E}_{T}$ should not be substantially reduced. This analysis is also sensitive to another possible scenario, proposed in [13], in which a light axion that decays into muon pairs takes the place of the dark photon in the decays described above.

Data for this analysis correspond to an integrated luminosity of $4.1 \mathrm{fb}^{-1}$ after application of data quality and trigger requirements. Events must satisfy a set of high transverse energy $\left(E_{T}\right)$, single electromagnetic $(\mathrm{EM})$ cluster triggers which are fully efficient for photons with $E_{T}>$ $30 \mathrm{GeV}$.

EM clusters are selected from calorimeter clusters, built using the simple cone algorithm of radius $\mathcal{R}=$ $\sqrt{(\Delta \eta)^{2}+(\Delta \phi)^{2}}=0.4$ [14], by requiring that the fraction of the energy deposited in the EM section of the calorimeter, $\mathrm{EM}_{\text {frac }}$, is above $95 \%$ and the calorimeter isolation variable $I=\left[E_{\mathrm{tot}}(0.4)-E_{\mathrm{EM}}(0.2)\right] / E_{\mathrm{EM}}(0.2)$ is less than 0.2 , where $E_{\mathrm{tot}}(0.4)$ is the total energy in a cone of radius $\mathcal{R}=0.4$, corrected for the underlying event contribution, and $E_{\mathrm{EM}}(0.2)$ is the EM energy in a cone of radius $\mathcal{R}=0.2$, which is taken to be the EM cluster energy.

Photon candidates are selected from central calorimeter $(|\eta|<1.1)$ EM clusters which have (i) $\mathrm{EM}_{\text {frac }}>97 \%$, 
(ii) $I<0.07$, (iii) a shower shape consistent with that of a photon, and (iv) the scalar sum of the transverse momenta $\left(p_{T}\right)$ of all tracks originating from the primary vertex in an annulus $0.05<\mathcal{R}<0.4$ around the cluster less than $2 \mathrm{GeV}$. Additionally, we require that photon candidates are not spatially matched to activity in the tracker. The tracker activity can either be a reconstructed charged particle's track or a density of hits in the silicon microstrip and central fiber trackers consistent with a track. The EM clusters that do not have matched activity in the tracker, but fail other photon selection criteria, are dominated by jets that have fragmented into neutral pions, and are referred to below as fake photons.

We search for dark photons in events with at least one photon with $E_{T}>30 \mathrm{GeV}$ and $\mathbb{E}_{T}>20 \mathrm{GeV}\left(\mathscr{E}_{T}\right.$ is computed using all calorimeter cells and corrected for EM and jet energy scales). Dark photon candidates are formed by selecting pairs of oppositely charged spatially close $(\mathcal{R}<$ $0.2)$ tracks that originate from the same point $(|\Delta z|<$ $2 \mathrm{~cm}$ ) along the beam line. The leading (trailing) track $p_{T}$ is required to exceed 10 (5) $\mathrm{GeV}$, which is highly efficient for the signal and suppresses the multijet background. We then require the scalar sum of $p_{T}$ of all tracks excluding the pair in a cone of radius 0.4 centered on the pair momentum direction to be less than $2 \mathrm{GeV}$. To further reduce the multijet background we require that each track must have its azimuthal angle not aligned with a photon, $0.4<\Delta \phi_{\gamma \text {,track }}<2.74$. In rare cases, when there is more than one such pair in the event, we select the one with the highest trailing track $p_{T}$.

For a dark photon decaying into a pair of electrons, the calorimeter depositions overlap, so we require that the dark photon candidate matches an EM cluster with $E_{T}>$ $10 \mathrm{GeV}, \mathrm{EM}_{\text {frac }}>97 \%$, and $I<0.1$. For the dimuon decay mode, we require that at least one of the tracks is matched to a reconstructed muon, and the energy deposited in the calorimeter in the annulus $0.1<\mathcal{R}<0.4$ is below $3 \mathrm{GeV}$.

Dark photons would manifest themselves as a narrow peak in the lepton pair invariant mass distribution. We use a Monte Carlo simulation to characterize the mass resolution, as well as the efficiency to reconstruct the events. SUSYHIT [15] is used to calculate masses and decay probabilities for the SUSY [16] model, known as Snowmass Slope SPS 8, and produce the Les Houches Accord [17] card files. These files are modified to introduce neutralino decays to a dark photon. Events with one of the two neutralinos decaying into a dark photon and the other decaying into a photon are generated with PYTHIA [18] using CTEQ6L1 parton distributions [19] and are passed through the full GEANT-based [20] detector simulation and the same reconstruction chain as the data. Following [21], the leading order (LO) signal cross sections calculated by PYTHIA are scaled to match the next-to-leading order (NLO) prediction using $k$-factor values extracted from [22]. The event kinematics depends on the mass of the dark photon and the masses of superpartners, resulting in variations in selection efficiency between $5 \%$ and $12 \%$.

There are three types of SM processes that contribute to our data sample: (B1) QCD events with real or fake photons and mismeasured $\mathbb{E}_{T}$. These contain jets or photon conversions faking the dark photon. (B2) $W \rightarrow e / \mu \nu$ plus a real or fake photon. The dark photon is faked by a accidental overlap of a high $p_{T}$ track with the lepton. (B3) $W \rightarrow \tau \nu \rightarrow 3 h^{ \pm} \nu$ plus real or fake photon. One of the particles from $\tau$ lepton decays is lost or very soft, and the remaining $\tau$ decay products fake the dark photon.

We study dark photon candidate mass distributions in three control samples where we do not expect dark photons to appear. The $\mathrm{QCD}_{\gamma}$ control sample is selected by reversing the $\mathscr{E}_{T}$ cut. The $\mathrm{QCD}_{\text {jet }}$ sample is selected by using the same criteria as the $\mathrm{QCD}_{\gamma}$ sample, but requiring a fake photon instead of a photon. Finally, the $\mathrm{QCD}_{W}$ sample requires a fake photon and $\mathbb{E}_{T}>20 \mathrm{GeV}$. All three have contributions from B1, although the relative fraction of multijets, single photon production, and diphoton production varies among the three control samples. Backgrounds B2 and B3, however, can only significantly contribute to the $\mathrm{QCD}_{W}$ sample. We observe no difference between the dark photon candidate mass distributions in the three control samples. We therefore conclude that the background to dark photon production is dominated by B1, and use the average shape of the dark photon candidates mass distributions in all control samples as our background model.

The dark photon candidate invariant mass distributions in the signal sample are shown in Fig. 2 separately for the electron and muon channels, together with the expected contribution from dark photons with a mass of $1.4 \mathrm{GeV}$.

We see no evidence of a dark photon signal and proceed to set limits on its production. To set limits we use the standard D0 likelihood fitter [23] that incorporates a log-
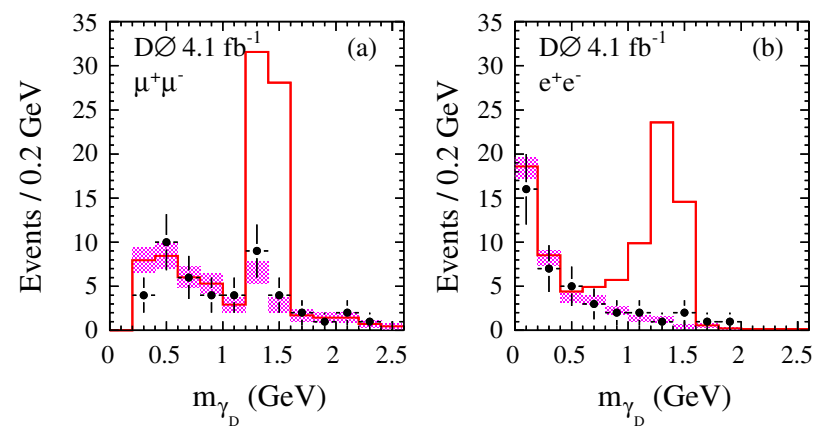

FIG. 2 (color online). Observed mass distributions in the signal region are represented as points with error bars, the background estimation is shown as filled band, and an example signal for $m_{\gamma_{D}}=1.4 \mathrm{GeV}$ plus background is shown as the solid histogram for the dimuon channel (a) and the dielectron channel (b). 


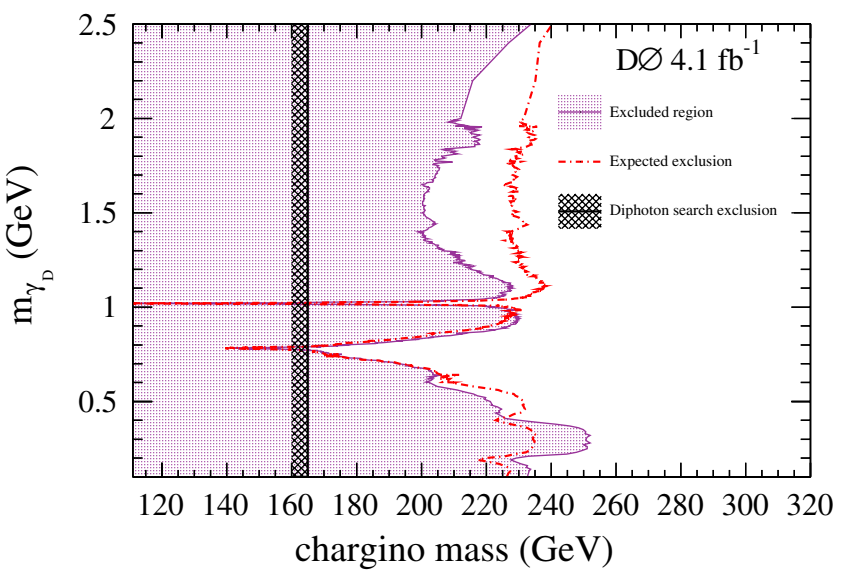

FIG. 3 (color online). The excluded region of possible masses of the lightest chargino and the dark photon for $\mathcal{B}=0.5$ are shown as the shaded region. The expected limit is illustrated as the dash-dotted line. The vertical black line corresponds to the exclusion from the diphoton search [21].

likelihood ratio (LLR) statistic method [24]. The value of $C L_{s}$ is defined as $C L_{s}=C L_{s+b} / C L_{b}$, where $C L_{s+b}$ and $C L_{b}$ are the confidence levels for the signal plus background hypothesis and the background-only (null) hypothesis, respectively. These confidence levels are evaluated by integrating the corresponding LLR distribution populated by simulating outcomes via Poisson statistics. Systematic uncertainties are treated as uncertainties on the expected number of signal and background events, not the outcomes of the limit calculations. This approach ensures that the uncertainties and their correlations are propagated to the outcome with their proper weights. The limit is set by simultaneously fitting dilepton invariant mass distributions in data for the muon and electron channels to the signal and background predictions for each

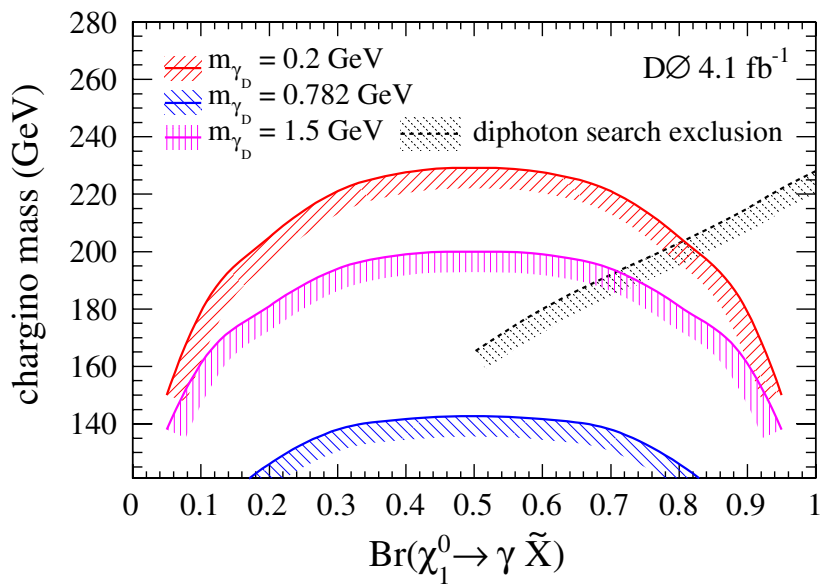

FIG. 4 (color online). The dependence of the excluded chargino masses on the branching ratio of the neutralino into a photon are given for dark photon masses of $0.2,0.782$, and $1.5 \mathrm{GeV}$. The black contour corresponds to the exclusion from the diphoton search [21]. signal point, defined by the dark photon and the lightest chargino masses. For each dark photon mass the background is normalized outside of the expected signal region. The systematic uncertainty on the signal reconstruction efficiency $(25 \%)$ is dominated by the uncertainty to reconstruct the two spatially close tracks from the dark photon decays (20\%). This efficiency varies from $70 \%$ to $95 \%$ depending on the opening angle between the tracks, and was validated with data using tau decays and converted photons in radiative $Z \rightarrow \mu \mu \gamma$ decays. We also took into account the uncertainty on the total integrated luminosity $(6.1 \%)$ and the effect of varying the dark photon mass resolution by $10 \%$.

We interpret the cross section limits as limits on the lightest chargino mass as a function of the dark photon mass and the neutralino branching fraction. For $\mathcal{B}=0.5$ the excluded region of chargino and dark photon masses is shown in Fig. 3. The difference between the observed and expected limits never exceeds 2 standard deviations over the whole dark photon mass range. In Fig. 4 we display the chargino mass limit as a function of $\mathcal{B}$ for three representative dark photon masses: $0.2 \mathrm{GeV}$ (only the electron channel is open), $0.782 \mathrm{GeV}$ (low branching fraction into leptons due to $\omega$ and $\rho$ mesons), and $1.5 \mathrm{GeV}$. Our previous limit on the SUSY in the diphoton final state [21] is directly applicable to the model considered in this Letter, although it does not probe the dark photon mass. The corresponding exclusion contours are shown in Figs. 3 and 4.

To summarize, we search for a previously unexplored final state consisting of a photon, two spatially close leptons from hypothetical dark photon decays and large missing energy. We find no evidence for such events, and set limits on their production in a benchmark model [10]. For dark photon masses of $0.2,0.782$, and $1.5 \mathrm{GeV}$ we exclude chargino masses of 230,142, and $200 \mathrm{GeV}$, respectively.

We would like to thank Scott Thomas and David Shih for many inspiring discussions and help with the signal simulation. We thank the staffs at Fermilab and collaborating institutions, and acknowledge support from the DOE and NSF (U.S.); CEA and CNRS/IN2P3 (France); FASI, Rosatom, and RFBR (Russia); CNPq, FAPERJ, FAPESP, and FUNDUNESP (Brazil); DAE and DST (India); Colciencias (Colombia); CONACyT (Mexico); KRF and KOSEF (Korea); CONICET and UBACyT (Argentina); FOM (The Netherlands); STFC and the Royal Society (United Kingdom); MSMT and GACR (Czech Republic); CRC Program, CFI, NSERC, and WestGrid Project (Canada); BMBF and DFG (Germany); SFI (Ireland); The Swedish Research Council (Sweden); CAS and CNSF (China); and the Alexander von Humboldt Foundation (Germany).

\footnotetext{
*Visitor from Augustana College, Sioux Falls, SD, USA.

${ }^{\dagger}$ Visitor from Rutgers University, Piscataway, NJ, USA.
} 
${ }^{\ddagger}$ Visitor from The University of Liverpool, Liverpool, United Kingdom.

${ }^{\S}$ Visitor from Centro de Investigacion en ComputacionIPN, Mexico City, Mexico.

"Visitor from ECFM, Universidad Autonoma de Sinaloa, Culiacán, Mexico.

"Visitor from Helsinki Institute of Physics, Helsinki, Finland.

**Visitor from Universität Bern, Bern, Switzerland.

${ }^{\dagger}$ Visitor from Universität Zürich, Zürich, Switzerland.

+ Deceased

[1] T. Han, Z. Si, K. Zurek, and M. Strassler, J. High Energy Phys. 07 (2008) 008; M. Strassler and K. Zurek, Phys. Lett. B 651, 374 (2007).

[2] G. R. Farrar and P. Fayet, Phys. Lett. B 79, 442 (1978).

[3] O. Adriani et al., Nature (London) 458, 607 (2009).

[4] J. Chang et al., Nature (London) 456, 362 (2008).

[5] A. A. Abdo et al., Phys. Rev. Lett. 102, 181101 (2009).

[6] N. Arkani-Hamed, D. P. Finkbeiner, T. R. Slatyer, and N. Weiner, Phys. Rev. D 79, 015014 (2009); N. ArkaniHamed and N. Weiner, J. High Energy Phys. 12 (2008) 104.

[7] A. Sommerfeld, Ann. Phys. (Leipzig) 403, 257 (1931).

[8] R. Bernabi et al. (DAMA/LIBRA Collaboration), Eur. Phys. J. C 56, 333 (2008).

[9] C. Amsler et al., Phys. Lett. B 667, 1 (2008).

[10] D. Shih and S. Thomas (to be published).

[11] P. Fayet, Phys. Lett. 70B, 461 (1977); 86B, 272 (1979); 175, 471 (1986); see also a review by G. F. Giudice and R. Rattazzi, in Perspectives on Supersymmetry, edited by G. L. Kane (World Scientific, Singapore, 1998), pp. 355377 , and references therein.
[12] V.M. Abazov et al. (D0 Collaboration), Nucl. Instrum. Methods Phys. Res., Sect. A 565, 463 (2006).

[13] Y. Nomura and J. Thaler, Phys. Rev. D 79, 075008 (2009).

[14] The D0 detector utilizes a right-handed coordinate system with the $z$ axis pointing in the direction of the proton beam and the $y$ axis pointing upwards. The azimuthal angle $\phi$ is defined in the $x y$ plane measured from the $x$ axis. The pseudorapidity is defined as $\eta=-\ln [\tan (\theta / 2)]$, where $\theta=\arctan \left(\sqrt{x^{2}+y^{2}} / z\right)$.

[15] A. Djouadi, M. M. Mühlleitner, M. Spira, Acta Phys. Pol. B 38, 635 (2007).

[16] S. P. Martin, S. Moretti, J. M. Qian, and G. W. Wilson, in Proceedings of the APS/DPF/DPB Summer Study on the Future of Particle Physics (Snowmass 2001), edited by N. Graf, eConf C010630 (2001), p. 346; B. C. Allanach et al., Eur. Phys. J. C 25, 113 (2002).

[17] J. Alwall et al., Comput. Phys. Commun. 176, 300 (2007).

[18] T. Sjöstrand et al., Comput. Phys. Commun. 135, 238 (2001).

[19] J. Pumplin et al., J. High Energy Phys. 07 (2002) 012; D. Stump et al., J. High Energy Phys. 10 (2003) 046.

[20] R. Brun and F. Carminati, CERN Program Library Long Writeup W5013, 1993 (unpublished).

[21] V. M. Abazov et al. (D0 Collaboration), Phys. Lett. B 659, 856 (2008)

[22] W. Beenakker et al., Phys. Rev. Lett. 83, 3780 (1999).

[23] W. Fisher, Report No. FERMILAB-TM-2386-E.

[24] T. Junk, Nucl. Instrum. Methods Phys. Res., Sect. A 434, 435 (1999); A. Read, Report No. CERN 2000-005, 2000. 